\title{
Más allá de las dicotomías. Un análisis de la actividad del trenzado en la diáspora senegalesa desde el feminismo negro/Beyond Dicotomies. An
}

\section{Analysis of the Braided Activity in the Senegalese Diaspora since Black Feminism}

\author{
Mercedes Jabardo Velasco \\ Universidad Miguel Hernández (UHM). España/Spain \\ jabardo@umh.es
}

\section{*Beatriz Ródenas Cerezo}

Instituto de Estudios del Bajo Cinca, Centro de Estudios de Monzón y Cinca Medio, Centro de Estudios del Somontano de Barbastro (IEBC; Cehimo; CESB). España/Spain

rodenasb@hotmail.com

Recibido / Received: 30/11/2016

Aceptado / Accepted: 23/01/2017

\section{RESUMEN}

Nuestro objetivo en este texto es situar en la centralidad el espacio femenino, y probarlo como categoría analítica en el marco de los debates que articulan movilidades, género y generaciones, aplicándolo al análisis de la migración femenina de origen senegalés en España. Centrándonos en una actividad económica como el trenzado, pretendemos desligar el debate en torno al emprendimiento femenino y la agencia de las mujeres migrantes en contextos transnacionales, de las lógicas que lo identifican con la "conquista" del espacio público. Localizando en el espacio femenino las dinámicas desde donde se reproducen, se resisten 0 transforman las relaciones de género, incorporamos éste como locus de enunciación y forzamos el cuestionamiento de la dicotomía público/privado, recuperando los cuestionamientos dicotómicos que se han planteado desde el feminismo negro.

Palabras clave: Feminismo negro, trenzado, espacio femenino, movilidades, Senegal.

\section{ABSTRACT}

Our objective in this text is to situate the feminine space in the centrality, and to test it as an analytical category within the framework of the debates that articulate mobilities, gender and generations, applying it to the analysis of female migration of Senegalese origin in Spain. Focusing on an economic activity such as braiding, we intend to disconnect the debate about female entrepreneurship and the agency of migrant women in transnational contexts, from the logics that identify it with the "conquest" of public space. Locating in the feminine space the dynamics from which gender relations are reproduced, resisted or transformed, we incorporate this as locus of enunciation and we force the questioning of the public / private dichotomy, recovering the dichotomous questionings that have been raised since black feminism.

Key words: Black feminism, braided, feminine sphere, mobilities, Senegal.

\footnotetext{
*Autor para correspondencia / Corresponding author: Mercedes Jabardo Velasco. Plaza Abad Penalva, 1 Ent. Izq. 03002. Alicante y Beatriz Ródenas Cerezo. Avda. Pirineos, 2, 3. ${ }^{\circ} \mathrm{K} 22430$ Graus (Huesca)

Sugerencia de cita / Suggested citation: Jabardo, M., Ródenas, B. (2017). Más allá de las dicotomías. Un análisis de la actividad del trenzado en la diáspora senegalesa desde el feminismo negro. Revista Española de Sociología, 26 (3), 373-384.
}

(doi:10.22325/fes/res.2017.29) 


\section{DESDE EL FEMINISMO NEGRO}

El pensamiento feminista negro ha interpelado las categorías desde donde se plantea la opresión/dependencia/emancipación de las mujeres en el feminismo hegemónico, muy en especial al concepto de género y las categorías desde donde se construye, tanto desde el feminismo liberal (público/privado) como desde el feminismo maxista (producción/reproducción). Las aportaciones más sugerentes han puesto en cuestión los conceptos que se han vuelto centrales en la teoría feminista: familia, reproducción y patriarcado. Y han definido líneas para construir una auténtica e inclusiva herstory incorporando las experiencias de las mujeres negras en una visión re-formulada de los tres conceptos (Jabardo, 2012). En este sentido fue especialmente sugerente la lectura de carácter transcultural que realizó Hazel Carby (2012) del sistema sexo-género (Rubin, 1986). Pluralizando el concepto planteó que cada relación familiar debía entenderse y explicarse desde la lógica propia de un sistema de sexo-género específico y cualquier patrón de subordinación debía ser historizado (Carby, 2012: 228).

Sin embargo, más estériles han sido los intentos de trascender las dicotomías. Probablemente porque no se ha puesto en cuestión el eje axial (de carácter dicotómico) desde donde se habla. En las múltiples referencias en relación a la posición de las mujeres negras en la dicotomía producción/ reproducción aparece claramente esta aporía. Se apuesta 'a pesar de su aparente simplicidad' por la deconstrucción del concepto de reproducción (Carby, 2012), pero no se altera la relación dicotómica entre producción/reproducción.

Tampoco el más reciente auge de la perspectiva interseccional (Hill Collins, 2000), que ha centrado las aportaciones teórico-metodológicas del feminismo negro a los estudios feministas y de género, ha ido en esa dirección. Ciertamente ha complejizado el análisis de la realidad vivida por las mujeres negras mediante el abordaje de las diferentes posicionalidades y las clasificaciones sociales, históricamente situadas ${ }^{1}$ (Magliano,

1 Existe un cierto consenso en torno al género, la raza, etnicidad, sexualidad y clase social como las clasificaciones sociales con mayor peso en la estructura de las relaciones sociales contemporáneas.
2015: 692). Lo cual ha dado pie a la recuperación de experiencias de éste (y otros grupos subordinados) al tiempo que se desentrañaban las relaciones de poder que enfrentan en diversos contextos socio-históricos. Pero lo han hecho sin abordar la cuestión que desde el pensamiento feminista negro se había planteado como central: el cuestionamiento de las categorías dicotómicas desde donde se piensa, eso que Audre Lorde llamó "el lenguaje del amo" (Citado en Jabardo, 2012: 33).

En este artículo nos proponemos recoger del feminismo negro británico el testigo del ejercicio deconstructivista de las diadas producción/reproducción y público/privado para tratar de trascenderlas, con el fin de adentrarnos en aquellas experiencias que aún hoy son producidas como no existentes (Santos, 2005) en los debates teóricos.

Pensar más allá de las dicotomías, desviar la mirada del reduccionismo al que éstas nos empujan, y revelar otras relaciones alternativas, supone un ejercicio de imaginación que el sociólogo B. S. Santos propone invitándonos a pensar acerca de los términos que las integran fuera de las articulaciones y relaciones de poder que los unen (Santos, 2005: 159). "¿Qué experiencias escapan a la dicotomía público/privado, producción/reproducción?", podríamos comenzar a plantear en una suerte de sociología de las ausencias (Santos, 2005: 160). Y añadir además: ¿qué fragmentos de la existencia y de las prácticas de las mujeres trans-migrantes de origen senegalés quedan fuera de la(s) relación(es) dicotómica(s)?

\section{EXPLORANDO EL DOMINIO FEMENINO: LÓGICAS DE SOLIDARIDAD Y COMPETENCIA}

Señala Eva Evers Rosander que las mujeres comerciantes senegalesas en Tenerife difuminando las fronteras entre el espacio doméstico y el espacio público consiguen mantener su reputación moral y decencia al tiempo que viajan solas: "extendiendo 0 estirando el contexto doméstico hasta incorporar también el espacio extranjero, disminuyen los riesgos de estar en la calle 0 en otros lugares dominados por los hombres" (Rosander, 2006: 119). También menciona la habilidad —más propia de mujeres que de los hombres- de integrar lo pú- 
blico y lo privado, lo profano con lo sagrado y lo moral con las realidades de la vida, como otro de los rasgos desde donde explicar —y justificar- la capacidad de las mismas mujeres para enfrentar las presiones de género. Constreñidas por la prevalencia de las ideologías de género que hacen más difícil su carrera transnacional, las comerciantes senegalesas - "cosmopolitas-locales" en términos de Rosander - son capaces de subvertirlas sin alejarse de los roles prescritos. "Mediante el trabajo duro, el éxito económico y su comportamiento moral cumplen las exigencias sobre ellas tanto en Senegal como en la diáspora... tendiendo puentes y buscando vías para una autonomía individual y relacional" (ibídem 129),

En una investigación más reciente sobre las comerciantes/emprendedoras senegalesas en Nueva York, Mariame S. Lo (2015) incide en las paradojas que encarnan, para las mujeres senegalesas, las redes sociales diaspóricas. "Representan un espacio transnacional económico habilitador, al tiempo que encarnan un espacio social informal que sin embargo mantiene sitios de luchas de poder profundamente insertados en dinámicas de género socioculturales y económicas que transfieren formas locales a contextos transnacionales" (Lo, 2015).

Ambas autoras dejan ver esos fragmentos de la existencia y de las prácticas de las mujeres transmigrantes senegalesas que no quedan recogidas bajo las categorías público/privado. Aunque ninguna habla explícitamente de un espacio intermedio, las dos fuerzan las categorías pre-existentes — "extensión del espacio doméstico" —dice Rosander; "espacio social informal", apunta Lo — con el objeto de incorporar dinámicas que solo pueden ser comprendidas en el marco de lo que Webner (1999) Ilama el "dominio culturalmente pre-establecido de poder femenino". Esta autora, que reflejó la capacidad de acción de las mujeres musulmanas en Londres - y sus luchas contra el fundamentalismo - en el escenario de la diáspora, situaba en el "dominio femenino" la "activa ciudadanía" de las mujeres en contextos marcados por estructuras de poder autoritarias, usualmente controladas por hombres (Webner, 1999). La reivindicación de los espacios femeninos como lugares de enunciación y, por tanto, como parte de la esfera pública (Benha- bib, 1992) ha desempeñado un papel importantísimo en la ampliación y complejización de los debates en torno a cuestiones relativas a la agencia de las mujeres en sociedades no occidentales (Mahmood, 2008), y han conseguido desviar la mirada del individualismo liberal hacia conceptos que sitúan la construcción de la subjetividad en clave relacional y/o social.

Partiendo de Sarah Mahmood y de su paradoja de la subjetivación (2008), podemos identificar las dinámicas desde donde se construye el espacio femenino en la sociedad senegalesa y su relectura en contextos de diáspora. "Si entendemos el poder como un conjunto de relaciones que no solo dominan al sujeto sino que crean las condiciones para su existencia... las condiciones y procesos que lo subordinan son los mismos que convierten al sujeto en consciente de sí mismo y en agente social", dice Mahmood (2008).

$Y$ es en ese contexto donde es posible entender las lógicas femeninas que dominan la actividad del trenzado:

"El trenzado es un mundo de mujeres. Donde se ayudan. Pero también compiten. Porque todas quieren lo mismo, que los turistas se detengan en su lugar, que sean ellas las elegidas" (Nagore: Alicante, 2016)

Ayuda y competencia son los dos términos que Nagore, una de nuestras interlocutoras senegalesas, asocia con el mundo de mujeres; y son los dos términos que trataremos de explicar para reflejar las lógicas del funcionamiento del espacio femenino, que presentaremos también como cultural e históricamente situado.

La institución de la poligamia nos permite ejemplificar y situar la paradoja de la subjetivización de la que nos habla Mahmood en el espacio femenino de la diáspora senegalesa; y comprender las lógicas de competencia/solidaridad que moldean las dinámicas del espacio femenino tanto en el contexto africano como en el espacio transnacional. Ambas lógicas forman parte de su proceso de socialización en un contexto marcado por unas ideologías de género que asignan a las mujeres un único rol. Las niñas crecen interiorizando el papel central que la maternidad tiene en su proceso de subjetivización y el imperativo moral de ejercerla dentro de la institución 
del matrimonio que, en Senegal, es culturalmente polígama. De hecho, aunque formal o jurídicamente se hable de distintos tipos de matrimonio (Dial, 2008), en la práctica hay multitud de estrategias para seguir manteniendo el sistema polígamo. Desde la presión social o mediática sobre las mujeres que permanecen solteras hasta esas otras pautas de comportamiento que las mujeres interiorizan desde que son niñas y que van minando su capacidad de resistencia a una institución que aceptan como "culturalmente impuesta". Nuestras informantes hablaban de la capacidad de aguantar. La socióloga y activista senegalesa Penda Mbow habla del silenciamiento (de las emociones) y de la desconfianza (primero hacia la co-esposa, después a cualquiera que pudiera llegar a serlo) (Mbow, 2011 citado en Rosander, 2016). Por otra parte es su propia vulnerabilidad en el terreno de la conyugabilidad el motor que guía sus alianzas con otras mujeres, y mueve sus resortes para posicionarse económica y socialmente en el "dominio femenino". La extraordinaria habilidad de las mujeres senegalesas para organizarse forma parte también de su capital humano, un recurso que han ido adquiriendo desde la infancia, y que han ido aprendiendo tanto en los hogares como en sus asociaciones juveniles (Jabardo, 2006).

Todo ello nos lleva a afirmar que la poligamia en tanto que institución sitúa a las mujeres en posiciones diferentes: de vulnerabilidad en el marco de las relaciones conyugales, de competitividad en el espacio doméstico y de solidaridad/competitividad en el espacio femenino. Y nos permite mostrar la capacidad de las mujeres para crear sus propias redes $-y$ la manera asombrosa en la que cuidan e invierten en las mismas - tanto como reflejo de la posición desigual donde el sistema las coloca cuanto estrategia/ fuente de poder para subvertir (o no) su posición en tanto que miembr(a) de un grupo subyugado.

\section{APUNTES METODOLÓGICOS}

Nuestros referentes etnográficos se localizan en la Comunidad Valenciana, particularmente en las ciudades de Alicante y Valencia, donde el trenzado se ha ido consolidando como un nicho étnico generizado y donde podemos explorar la complejidad de la construcción de subjetividades entre las mujeres senegalesas de dos generaciones (una antigua y una reciente migración femenina) que se mueven entre la tensión normativa de la comunidad en la que se anclan y los recursos que han ido adquiriendo en las rutas por las que han ido transitando.

El trabajo de campo se realizó en dos periodos: uno intensivo, entre los meses de junio-septiembre de 2010 y otro intermitente, entre mayo de 2014 y julio de 2015. Se combinaron diferentes técnicas de investigación: observación participante, entrevistas abiertas y confección de historias de vida. En un primer momento, las entrevistas semiestructuradas sirvieron a las investigadoras como método exploratorio, a través del cual detectar los temas recurrentes entre las entrevistadas, y cubrir los objetivos de la investigación, que venían dados por el planteamiento teórico inicial: situar el trenzado dentro de los debates teóricos actuales, incorporando un espacio femenino con sus propias lógicas que cuestionara desde la perspectiva de los feminismos negros las dicotomías público/privado y producción/reproducción.

Las diferentes entrevistas realizadas dieron lugar a la confección de 15 historias de vida en total, que permitieron conocer el punto de vista de las trenzadoras senegalesas. Confeccionar historias de vida como técnica etnográfica supone ya un análisis previo de los temas surgidos en las entrevistas, de los guiones producidos en las narrativas de cada mujer, y del contexto que envuelve a las entrevistas realizadas, poniendo en relación investigadora e informadora. Por esta razón al seguir el enfoque biográfico, tal y como lo inauguró la Escuela de Chicago, cada documento surgido de cada historia de vida supone un hecho único, e irremplazable, ya que permite producir un tipo de información que difícilmente puede producirse a través de otro tipo de técnicas.

En el texto que sigue, se han utilizado diferentes segmentos de las historias de vida con una intención analítica y expresiva (Bertaux, 1989). Al presentar diferentes segmentos de las narraciones recogidas se pretende comparar casos de mujeres ubicadas en contextos socioeconómicos diferentes, a la vez que ejemplificar los resultados del análisis realizado. Somos conscientes de que con el simple 
uso de la cita no se transmite la visión de las actrices, sino la interpretación de las investigadoras, por este motivo se deben ubicar las narraciones en el contexto teórico del que partimos, y sin perder de vista los objetivos que perseguimos.

\section{INTRODUCIENDO EL TRENZADO}

Coincidiendo con el milenio, una nueva literatura está sustituyendo a la que hasta finales del siglo pasado, seguía focalizando el fenómeno de la migración senegalesa en las actividades comerciales de carácter transnacional protagonizadas por varones y su vinculación con las cofradías religiosas, muy especialmente la cofradía mouride. Son ya varios los estudios que están visibilizando un nuevo perfil: mujeres emprendedoras que están practicando un nuevo cosmopolitismo vernacular (Diouf, 2000) en base a la actividad comercial y empresarial en escenarios transnacionales (Babou, 2008; Kane, 2013; Lo, 2015). Como planteó Diouf para el caso de los modou-modou, esas nuevas "cosmopolitas locales" (por utilizar la acertada expresión de Rosander) están explorando nuevos nichos en la economía global desarrollando habilidades y destrezas de carácter local (Babou, 2008; Lo, 2015). La proliferación de peluquerías en los centros urbanos con mayor presencia de inmigración africana que, por un lado, cuidan el pelo afro y por otro, son un reclamo de carácter étnico para un público más mayoritario ávido de consumir bienes culturales (Petronoti, 2010), es una muestra de ello. Como Babou ha señalado: "hair dressing was primarily a domestic activity of women, an important dimension of female sociability. Family members and close friends did each other's hair, and the long hours that women spent together dressing their hair provided time for gossips and social interactions. The knowledge of the art of grooming hair was passed down from grandmothers to mothers and daughters; knowing how to take care of one's daughters' hair was an integral part of motherhood" (Babou, 2008: 4). Lo que era una actividad doméstica en contexto africano, se ha transformado en una de las más importantes fuentes de ingresos para las mujeres senegalesas en contexto transna- cional (Babou, 2008). La aparición de peluquerías y la inserción de las mujeres dentro de la economía formal solo es una de ellas. Más relevancia ha tenido - aunque menos visibilidad en los debates teóricos - la actividad de trenzado que las migrantes transnacionales de origen senegalés han realizado al margen de la "economía formal".

Rosander lo reflejó en trabajos pioneros sobre la migración femenina de origen senegalés en las Islas Canarias. En un periodo en el que todavía era insignificante la presencia de mujeres entre los senegaleses asentados en España, identificó el primer flujo de mujeres migrantes autónomas en uno de los principales enclaves turísticos de la geografía española (Jabardo, 2006), así como su habilidad a la hora de movilizar un recurso de carácter étnico - su destreza como trenzadoras - para crear un nuevo nicho económico al margen del mercado de trabajo general y fuera del espacio público (Rosander, 2006). El hecho de que la mayoría de las que se asentaron en Canarias fueran de origen griotte, un grupo de bajo estatus en la rígida estructura social senegalesa, no es un dato baladí. La actividad del trenzado - así como todas las que tienen relación con el cuidado del pelo — eran actividades que se realizaban dentro del espacio doméstico. Fuera de los círculos de relación, el cuidado del pelo era una actividad socialmente desvalorizada, solo propia de mujeres de ese origen. Así como también eran propias del grupo griotte las habilidades que las mujeres senegalesas desplegaron a la hora de comercializar el trenzado como souvenir turístico: destreza en la negociación y habilidad para construir relatos y loas que aprovecharon para atraer y retener a las turistas. Ello junto a la capacidad de ahorro utilizando recursos de sistema de ahorro alternativos (como las tontines) que bien podríamos considerar como recurso y/o capital de género, permitió a las mujeres senegalesas ganar bastante dinero. Tanto como para poder incluso invertir en prestigio (Rosander, 2010).

Nuestras aportaciones etnográficas a este debate pretenden reflejar, en primer lugar, cómo una actividad en principio socialmente desvalorizada se revaloriza en contexto migratorio y se incorpora como parte del capital humano que portan las mujeres en la aventura migratoria; $y$, en segundo lugar, 
cómo en este espacio (necesariamente competitivo) operan las lógicas del espacio femenino.

Centrarnos en sendos enclaves turísticos de la Comunidad Valenciana como Valencia y Alicante nos permite comprobar hasta qué punto el trenzado se ha consolidado como un "nicho étnico generizado", en tanto que desempeña un papel semejante al que representa la actividad comercial entre los varones senegaleses: como un espacio en el que se integran las recién llegadas y al que se acude cuando falla el mercado de trabajo general.

\section{EL TRENZADO COMO NICHO ÉTNICO GENERIZADO}

La reunión de las trenzadoras en un mismo lugar, al mismo tiempo, crea diferentes juegos de solidaridad y competencia que van dibujando las redes sociales donde se ubican las senegalesas, según sus diferentes perfiles. Basándonos en los datos etnográficos que poseemos, vamos a reconstruir tres rutas migratorias de mujeres que han ocupado el nicho del trenzado como actividad estival: Aisa y Awa como mujeres reagrupadas; Ami y Maimuna que llegaron solas, Ami, contratada en origen, Maimuna, con visado de turista; y a través de cadenas migratorias femeninas expondremos los casos de Khady y Penda, que trabajan juntas, y Binta. Señalaremos, además, cómo utilizan el dinero recaudado con las trenzas, lo que, como veremos, varía según el perfil poseído y el proyecto migratorio que portan en la maleta.

Aisa es una de las pioneras. Llegó a Alicante en 1994, cuando su marido la reagrupó. En España, Aisa se hizo cocinera y durante años trabajó en la hostelería, hasta que la crisis la expulsó del sector.

Desde que se instaló la crisis —reflexiona Aisa - no hay trabajo para una negra en la hostelería. Cada vez que he intentado recuperar mi trabajo, o conseguir uno nuevo en el sector, me dicen que ahora hay muchos españoles en paro y que estaría muy mal visto que dieran un trabajo a una extranjera habiendo españoles en paro.

Aisa subraya más su identidad como negra que como senegalesa:

Aquí las cosas no van a cambiar - diceLo hemos intentado con Elena, contigo... pero no va a ser posible traspasar la línea del racismo. Y eso te hace mucho daño. Yo he estado muy deprimida, pequeña... porque cuando comienzas a verte en los ojos de la gente hasta tu postura corporal cambia. Ahora ya estoy mejor. He decidido ocuparme de lo mío. Hago trenzas, puedo desarrollarme desde la "africanidad" (soy yo quien termina colocando estas palabras para tratar de reflejar lo que estamos diciendo) y no hay problema. Ese espacio —el mío, el que se supone que me pertenece- me lo dejan.

Sí, es cierto que Aisa se encuentra muy cómoda ahí, en el paseo del puerto. Distante del resto de las mujeres africanas. No solo en experiencia, formación, bagaje, recorrido... también en las formas. No está para competir con ninguna para hacerse con la clientela. Está alejada, al lado de los vendedores, ofreciendo su mercancía. Por ahí transita menos público, pero el que llega lo intercepta, con una sonrisa, una invitación... sin moverse.

Awa tiene 34 años y llegó reagrupada por su marido en 2006. Es segunda co-esposa, la primera está en Senegal, y tiene dos hijas, una en Senegal y otra viviendo con ellos en Valencia. Cuenta que cuando llegó, con los papeles de la reagrupación no podía trabajar, y después tuvo a su hija:

Lo que pasa es que la niña no me deja trabajar... por eso yo quiero tirarla a mi país... se lo digo y dice, no, yo no quiero irme, yo quedarme aquí... Yo hace 304 años que tengo papeles para trabajar, pero nunca he encontrado trabajo... me voy a la playa a hacer trenzas, el mes de julio, hasta septiembre...

Awa prosigue:

Yo trabajaba en verano con las trenzas, y ganaba dinero, pero éste no es dinero de verdad. El dinero de las trenzas siempre se me va y no puedo comprar un billete a Senegal. En tres años que he trabajado con las trenzas, siempre ha pasado algo que se ha llevado mi dinero. Primero me llamó mi madre que estaba mala, después se puso malo mi hermano, y después mi marido dejó de cobrar subsidio... ahora llevo un mes trabajando, y le he dicho a mi marido que tengo que comprar un billete... este mes no digo nada, pero el siguiente... sino no voy a cocinar, no me voy a duchar... ríe... 
Ami es una joven de 29 años que llegó a España en 2008 como parte de un contingente laboral contratado en origen:

Cuando acabé, fui a Palma de Mallorca, a vender, y después aquí (a Valencia). Aquí no tengo familia, pero un amigo me dijo que las cosas estaban bien.

Ami cuenta que no tiene familia en España. Que está casada pero no tiene hijos, y su marido está en Senegal, trabajando.

Ami relata:

Ahora no estoy allí [Mallorca] porque es un pueblo, y no hay nadie, no puedes salir y a mí me gusta mucho hablar... Allí vivo en una casa con dos chicas más y tres chicos. Alquilamos un apartamento..., una amiga que está en Almería, otra que está en Mallorca, pagamos 120 euros cada una... Y la chica de allí me pide 20 euros todas las semanas para comprar comida, y eso es mucho... Ella quería venir aquí, conmigo, pero quería que buscáramos una casa bien para las dos... y yo no puedo gastar tanto... ahora vivo en una habitación alquilada, con pakistaníes. Antes, en Palma de Mallorca, vivía con una familia senegalesa que me trató muy mal. Dormía en un sofá, cocinaba, limpiaba, fregaba, planchaba, iba a comprar... no comía con ellos ni me pagaban. Aguanté así varios meses. Su hijo fue a Senegal por el cordero y yo le di 400 euros para que le diera a mi madre, y se lo quedó. Al final me fui. Discutí con ella, y ella decía que yo no era nadie para hablarle, que ella llevaba muchos años aquí, que si quería, hablara con mi madre, y mi madre le dijera a ella lo que sea. Luego dijo que yo le había insultado... ahora nos vemos en Palma y no nos hablamos, ni nos saludamos... pero desde que se comió mi dinero las cosas le van mal...

Ami continúa:

Allí [Mallorca] se gana mucho dinero y nos lo pasamos muy bien... Y las trenzas se cobran muy caras, 1800 más todo el pelo. Pero hay muchos problemas con la policía... siempre correr... Al final conocía a algunos, me llaman por mi nombre y todo, me dicen 'tú me tomas el pelo'...
En cuanto al destino que le da al dinero que gana, responde:

Mi padre murió, nosotros somos 4 hermanos y dos hermanas, además de los hijos de la otra esposa de mi padre... así, no tengo que mandar dinero todos los meses, sólo cuando puedo... compré una nevera a mi madre para que vendiera hielo...

Además, cuenta que ha ahorrado $1500 €$ para pagar los gastos de un contrato y pedir el arraigo social.

Maimuna también alimentó el sueño migratorio desde Senegal. Su hermano, que ahora reside en Alemania, y su primo, ya instalado en España, hablaban de las ventajas de vivir en un país europeo:

Recuerdo — decía - que lo primero que me dijo mi hermano es que aquí tenía un médico para él solo.

Pero solo decidió emigrar cuando, divorciada, fue víctima del ostracismo social. Antes, en Senegal, tenía una vida, un trabajo como profesora, una hija, un marido con buena posición económica. Llegó a España en el año 2009, "de vacaciones", que es como viajan quienes han conseguido un visado. Y se instaló en Alicante, junto a un familiar. Entre la comunidad de senegaleses encontró a su actual marido. Con él ha tenido a su segundo hijo, Mohamed. Me dice que ella nunca había hecho trenzas, ni siquiera a sus hermanas.

Al principio lo pasé mal. Con las mujeres hay que encontrar el lugar. Descubrir qué puedes hablar con gente con la que en principio no tienes nada en común. Y es duro. Porque ni ellas me entendían ni yo sabía cómo comportarme con ellas... Pero ahora es diferente. Ahora me respetan, y me han hecho un hueco. En este tiempo me he dado cuenta de que mi cultura, mi formación, me sirve (también en las trenzas). La manera en la que me relaciono, la forma en la que hablo... atrae a los clientes. Hay mucha gente que viene por algo más que las trenzas, que buscan conversación, que quieren - a través de las trenzas - entrar en nuestra cultura. Y yo puedo hablar con ellos. Y esta forma de estar me da prestigio entre las mujeres". "Yo sé por qué te respetan" —le digo. "Te he visto actuar. Sé que eres generosa. Y sé que, al 
final, la generosidad es algo que se valora en la cultura senegalesa". "Es verdad" — confirma Maimuna-A veces, cuando viene alguien y se quiere comunicar, las chicas me buscan. Y yo dejo mi sitio, mi trabajo y me voy con ellas, y estoy con el cliente y le doy conversación y consigo el mejor precio para ellas; pienso en su negocio como si fuera el mío. Y ellas lo valoran. Y me lo devuelven en generosidad. Yo no soy muy buena con las trenzas. Esto no es lo mío. Por eso cuando tengo algún cliente, vienen y me ayudan, y se ponen conmigo.

Khady y Penda trabajan juntas en verano y, explican, se reparten el dinero que ganan. Khady está divorciada y mantiene a dos niñas en Senegal. Penda es soltera y sin hijos, y se va a casar con un senegalés que ha conocido en Valencia, quien mantiene a su primera esposa en Senegal. Ambas llegaron a través de cadenas migratorias femeninas.

Khady y Penda aprovechan los festejos estivales para viajar por todo el país haciendo trenzas. Penda cuenta:

Ayer volvimos de San Sebastián y mañana nos vamos a Galicia... a veces dormimos en los coches de los senegaleses... También nos vamos a Teruel (al Torico)... a dormir en la calle... queríamos ir a Pamplona (San Fermín) donde Rama tiene una amiga, pero está más lejos y la amiga de Rama dice que hay mucha policía...

Penda sigue trenzando en invierno, además de realizar otras actividades:

En verano hago trenzas en la playa... y también cuando hay bodas, bautizos... me Ilaman. En invierno tengo collares...

En cuanto al destino del dinero, contesta:

Yo mando dinero a mi madre y hermanos, pero solo cuando puedo, en verano.

Khady también cuenta su rutina veraniega:

Este verano estuve en Bilbao, yo sola. Allí las chicas se compran una tienda de campaña y duermes ahí, pero este año me voy a quedar en Malvarrosa mejor, porque en Bilbao ganas 40 pero gastas 30 en comer, ducharte, lavarte, dormir... En Malvarrosa ganas 20 ¿qué es mejor? Si el año que viene Rama está en Puerto de Sagunto, mucho mejor... en puerto de Sagunto es mucho mejor, menos policía y más dinero, pero el mercado no lo abren hasta las 7 y el último autobús sale a las $9 \ldots$ si no tienes coche no puedes ir..." En invierno Khady realiza varios trabajos, "en invierno vendo por los pueblos... ropa, como en Senegal. Después conseguí un trabajo en Alicante, cuidando a un anciano, y luego volví aquí (Valencia). Mientras no tenga trabajo seguiré en Valencia. También estuve en Tarragona, trabajando en un restaurante muy grande, pero pagaban muy mal... 3 euros la hora, y trabajas 15 horas diarias... 10 dejé, eso no es trabajo...

La diferencia entre Khady y Penda es que Khady sí debe mandar dinero para sus hijas que están en Senegal:

Mis hijas están en Senegal con mi madre. $Y_{0}$ tengo que mandar 800100 euros todos los meses, porque son mi responsabilidad, a mí no me gusta que mi madre se tenga que encargar de todo. Pero a veces no puedo...

Por último, Binta, llegó también a través de cadenas femeninas. Está divorciada y mantiene a dos niñas en Senegal. Binta llevaba tan sólo unos meses en Valencia cuando protagonizó en verano varias situaciones tensas con el resto de trenzadoras, ya que no quiso atenerse a los precios que marcaban el resto de chicas en la playa:

Binta tenía mucha cola, todo el mundo la esperaba a ella. Una clienta de la cola me dice que lo hacía más barato, "la primera me ha pedido 100 euros, la siguiente 80 , y la otra 60. Ella (Binta) me lo hace por 40. 100 euros es lo que me han pedido en la peluquería...". Binta contestaba, "estamos en crisis, hay que dar precios baratos... el pelo me cuesta 10 euros, si cobro 40 estoy ganando $30 . . . "$ La clienta continúa, "míralas allí", me señala con la cabeza al resto de trenzadoras de la playa, "desde que estoy aquí no se ha sentado nadie. Y mírala a ella (Binta) tiene cola". Le pregunto a Binta, ¿y las chicas no se enfadan contigo?, "sí... pero yo estoy buscándome la vida. Yo pregunto cuánto quieres pagar, ven, siéntate. Ellas no son mi padre, ni mis hermanas, ni mi madre. Yo no las conozco. Yo acabo y me voy a casa. Me da igual... el viernes estuve chi- 
llando con ella (señala a otra trenzadora con la cabeza)... Me decía que no podía cobrar sólo 5 euros, y yo le decía, es mi mano, es mi trabajo... Los senegaleses siempre igual, siempre hablando, bla, bla, Binta esto, bla, bla. Como no tienen trabajo se sientan y hablan. A mí no me gusta. Yo llego y saludo, pero despacio, con respeto, "hola Khady"... no me alegro, chillo... eso no me gusta. A mí no me gusta hablar de la gente... cuando estaba en Senegal salía a discotecas, mi madre, mi padre, nadie decía nada... aquí hablan... Binta va a discotecas, a mí no me gusta.

En cuanto al destino del dinero, Binta lleva muy poco tiempo en la ruta migratoria:

Ahora no tengo dinero para mandar a mis hijas... pero les mando regalitos cuando puedo.

En definitiva, los diferentes perfiles, rutas migratorias emprendidas y proyectos migratorios portados, se relacionan con las diferentes formas que tienen las senegalesas de utilizar la actividad del trenzado. En el caso de Ami, la ausencia de una red social tupida en su ruta migratoria la llevó hacia uno de los enclaves turísticos más densos del país (Baleares), y, como consecuencia, ha vivido de cerca las tensiones de la competencia entre trenzadoras. Ami trabaja en verano en Mallorca consiguiendo ingresos que le cubren todo el año, lo que puede permitirse debido a la relativa ausencia de obligaciones y responsabilidades en su familia de origen. En su caso, Maimuna, representa cómo se ponen en juego destrezas y habilidades híbridas/ mestizas diferentes al recurso del trenzado, pero, sin embargo, es capaz también de utilizar el nicho del trenzado usando estas habilidades, y poniéndolas en valor a través del juego cooperación-competencia con otras mujeres.

Aisa y Awa nos muestran cómo las mujeres senegalesas se vuelven hacia el trenzado cuando el mercado falla y las dinámicas político-económicas las expulsan de él. Aisa pone de manifiesto su africanidad para explicar su situación, Awa habla de su posición como esposa reagrupada y como mujer que ejerce la maternidad a tiempo completo. Para Awa el trenzado representa una vía (prácticamente la única que ha tenido) para conseguir ingresos al margen de su marido, los cuáles ha utilizado para reforzar su posición tanto en origen (tratando las "enfermedades" de su madre y de su hermano) como en destino (exigiendo ante su marido un billete para volver a Senegal). De esta manera, el fallo del mercado laboral al integrar a Awa, ha sido esquivado a través de poner en valor sus recursos de género en el nicho del trenzado.

Khady, Penda y Binta llegaron las tres a través de mujeres que hicieron de eslabón en cadenas migratorias diferentes. Penda posee un perfil muy distinto a las otras dos, es soltera, prácticamente sin obligaciones en origen, y a punto de casarse en Valencia. Además la red social que la envuelve le permite (a ella y a Khady) desplazarse por el país a través de contactos que mujeres asentadas - previamente reagrupadas - han establecido con anterioridad (Rama). Sin embargo Khady porta un peso adicional, la obligación de mandar dinero a sus dos hijas en Senegal, peso que Khady sobrelleva intentando trabajar también en invierno, y acudiendo a lugares turísticos con menos competencia (Bilbao), en un intento de ganar más dinero con las trenzas. Binta, que también porta el peso de sus dos hijas en origen, prefiere, no obstante, sacrificar la solidaridad potencial que puede conseguir en las redes sociales de Valencia, a costa de ganar más dinero con el trenzado. Binta lo hace de esta manera porque hace escasos meses que llegó a Valencia y apenas ha establecido contacto con una comunidad senegalesa que, además, la señala por su forma de actuar. Como consecuencia, Binta emigrará tras el verano hacia Vitoria, y ya no volverá a trabajar el trenzado en Valencia.

De esta manera podemos ver cómo las senegalesas han utilizado el trenzado a través de diferentes líneas de acción que varían según la diferente utilización de la solidaridad y la competencia que proporcionan los espacios femeninos. Pero sobre todo el trenzado nos permite ver cómo las mujeres senegalesas utilizan un recurso de género propio del ámbito doméstico, de formas diferentes, adaptándolo a los distintos contextos y según las diferentes necesidades de cada mujer. En consecuencia, las mujeres senegalesas toman un recurso propio del ámbito doméstico para ponerlo en valor en un espacio femenino como es el mercado laboral en contexto migratorio, ya sea de forma irregular, dando una oportunidad a 
mujeres que no tienen otras posibilidades laborales, como de forma regular, a través de peluquerías que en determinados asentamientos han prosperado de forma espectacular (Babou, 2008).

\section{CONCLUSIONES}

Este artículo se acerca al estudio de las movilidades de las mujeres senegalesas desde una perspectiva feminista negra. Con este objetivo se han recuperado algunos de los debates clásicos del feminismo negro británico de los años noventa en torno a las dicotomías público/privado y producción/reproducción y se ha explorado como categoría analítica ese espacio intermedio que sigue produciéndose como no existente en los estudios que se acercan a las movilidades con enfoque de género. Al romper la dicotomía público/doméstico situando la agencia de las mujeres más allá de la esfera doméstica, pero fuera de la esfera pública, se ha querido significar este espacio como "espacio femenino". Usualmente fuera de los textos que están visibilizando la feminización de la inmigración senegalesa, el espacio femenino se torna en este artículo en una categoría central. Más allá de la conquista del espacio público, que ilustran los textos sobre emprendimiento femenino senegalés, la actividad económica de muchas de las mujeres que han escogido la Comunidad Valenciana como lugar de residencia se desarrolla en un espacio donde imperan las lógicas del dominio femenino.

Centralizando el debate que está abriendo las categorías teóricas para incorporar las experiencias de las mujeres africanas en los relatos de la movilidad en Europa (Kron and Nieden, 2013) en una actividad en apariencia marginal como el trenzado en los enclaves turísticos del Mediterráneo español, se ha contribuido al reconocimiento del espacio femenino como locus de enunciación.

Haciendo uso de las narrativas de mujeres senegalesas de dos generaciones que depositan en la actividad del trenzado en el periodo actual sus expectativas de futuro, se ha introducido el espacio femenino como locus de cooperación y de competitividad. Entendiendo que las lógicas del espacio femenino deben ser historizadas y situadas, la ten- sión entre la solidaridad y desconfianza que operan en el espacio de la diáspora senegalesa, se ha analizado desde las ideologías de género y más específicamente a través de una institución como la poligamia que coloca a las mujeres en una posición de desigualdad frente a los hombres y en relación de competencia/solidaridad con las otras mujeres.

En este artículo también se ha reflejado la variabilidad y la complejidad en los procesos de construcción de subjetividades, difícilmente explicables en función de categorías dicotómicas que presentan la agencia en la tensión entre la tradición y la modernidad. Mostrar el espacio femenino como escenario de consentimiento, acomodación y/o resistencia permite reflejar la variabilidad de las estrategias de movilidad de las mujeres migrantes de origen africano y relacionarlas con las posiciones desde donde construyen sus subjetividades en el marco de unas ideologías de género que son o pueden ser tanto acatadas como interpeladas. Los procesos de mestizaje, hibridación que se muestran son ya un reflejo de la emergencia de otras "modernidades".

\section{AGRADECIMIENTOS Y FINANCIACIÓN}

Este artículo ha sido elaborado en el marco de investigaciones y debates surgidos en el seno del proyecto I+D+I "Género, transnacionalismo y estrategias intergeneracionales de movilidad social (FEM 2011-26210). Las autoras quisieran agradecer a Laura, Amelia y Almudena los comentarios al artículo; así como las lecturas que de él hicieron evaluadores externos.

\section{BIBLIOGRAFÍA}

Babou, C. A. (2008). Migration and cultural change: Money, "caste", gender, and social status among senegalese female hair braiders in the United States. Africa Today, 55 (2), 2-22.

Benhabib, S. (1992). Situating the Self. Gender, community and postmodernism in Contemporary Ethics. Cambridge: Polity Press.

Bertaux, D. (1989). Los relatos de vida en el análisis social. Historia y Fuente Oral, 1, 87-96. 
Carby, H. V. (2012) [1982]. Mujeres blancas, jescuchad! El feminismo negro y los límites de la hermandad femenina. En M. Jabardo (ed.) Feminismos negros. Una antología (pp. 209-243). Madrid: Traficantes de Sueños.

Collins, P. H. (2000). Black Feminist Thought. New York: Routledge.

Dial, F. D. (2008). Mariage et divorce à Dakar: itinéraires féminins. Dakar: Karthala.

Diouf, M. (2000). The Senegalese Murid Trade Diaspora and the Making of a Vernacular Cosmopolitanism. Public Culture, 12 (3), 679-702.

Jabardo, M. (2006). Senegaleses en España. Conexiones entre origen y destino. Madrid: Ministerio de Trabajo y Asuntos Sociales.

Jabardo, M. (2012). Feminismos negros. Una antología. Madrid: Traficantes de Sueños.

Jabardo, M. (2014). Ser africano en el Maresme. Migración, trabajo y etnicidad en la formación de un enclave étnico. Madrid: Última línea.

Kane, A. (2013). Introduction. En A. Kane, T. Leedy (eds.) African Migrations. Patterns and Perspectives. Bloomington and Indianapolis: Indiana University Press.

Kron, S., B. zur Nieden (2013). Thinking Beyond the Categories: on the Diasporization of Gender Studies. QJB-Querelles. Jahrbuch für Frauen-und Geschlechterforschung (en línea). http://www. querelles.de/index.php/qjb/article/view/1/2, acceso 15 de noviembre de 2015.

Lo, M. S. (2015). En route to New York: diasporic networks and the reconfiguration of female entrepreneurship in Senegal. Gender, Place \&Culture, 23 (4), 503-520.

Magliano, M. J. (2012). Interseccionalidad y migraciones: potencialidades y desafíos. Estudios feministas, Florianópolis, 23 (3), 691-712.

Mahmood, S. (2008). Teoría feminista y el agente social dócil: algunas reflexiones sobre el renacimiento islámico en Egipto. En L. Suárez Navaz y R. A. Hernández (eds.), Descolonizando el feminismo (pp. 165-221). Madrid: Cátedra.

Petronoti, Marina (2010). Weaving threads between the ethnic and the global: African women's entrepreneurial ventures in Athens. Anthropological Journal of European Cultures, 19 (2), 129-148.
Ródenas, B. (2014). Redes, matrimonio y agencia. Mujeres transnacionales entre Valencia y Senegal. Tesis de doctorado, Universidad Miguel Hernández.

Rosander, E. (2006). Cosmopolitas y Locales: mujeres senegalesas en movimiento. En M. Jabardo, Senegaleses en España. Conexiones entre origen y destino (pp. 117-129). Madrid: Ministerio de Trabajo y Asuntos Sociales.

Rosander, E. (2010). Gender Relations and female autonomy among Senegalese migrants in Spain: Three cases from Tenerife. African and Black Diaspora. 3 (1), 91-107.

Rosander, E. (2016). In Pursuit of Paradise. Senegalese Women, Muridism and Migration. Uppsala: The Nordic Africa Institute.

Rubin, G. (1986). El tráfico de mujeres: notas sobre la 'economía política' del sexo. Nueva Antropología, VIII, 30, 95-145.

Santos, B. S. (2005). El milenio huérfano. Madrid: Trotta.

Waldinger, R. (1994). The making of an Immigrant Niche. IMR, 28 (1), 3-30.

Werbner, P. (1999). Political motherhood and the feminization of citizenship: women's activisms and the transformation of the public sphere. En N. Yuval-Davis y P. Werbner (eds.) Women, Citizenship and Difference (pp. 221-245). London: Zed Books.

\section{NOTAS BIOGRÁFICAS}

Mercedes Jabardo Velasco, doctora en Antropología Social y licenciada en Filosofía por la Universidad Autónoma de Madrid. Profesora contratada doctora de Antropología Social en el departamento de Ciencias Sociales y Humanas de la Universidad Miguel Hernández de Elche. En la misma universidad es la directora del Máster oficial de investigación Nuevas Tendencias en Antropología Social. Escenarios de riesgo y estrategias de postdesarrollo, y coordinadora del programa de doctorado en Antropología Aplicada en contextos de crisis. Sus líneas de investigación: feminismos negros; género, migraciones y cultura; migraciones africanas y diáspora negra; movimientos sociales y postdesarrollo. Entre sus 
publicaciones: Senegaleses en España. Conexiones entre origen y destino (Madrid, Ministerio de Trabajo y Asuntos Sociales, 2006), Feminismos negros. Una antología (Madrid, Traficantes de Sueños, 2012) y Ser africano en el Maresme. Migración, trabajo y etnicidad en la formación de un enclave étnico (Madrid, Última Línea, 2014).

Beatriz Ródenas Cerezo, doctora en Antropología social por la Universidad Miguel Hernández y licenciada en Antropología social y cultural por la Universidad de Barcelona. Investigadora en el Instituto de Estudios del Bajo Cinca, en el Centro de Estudios de Monzón y Cinca Medio y en el Centro de Estudios de Somontano de Barbastro. Sus líneas de investigación: género, migraciones africanas, fenómenos de codesarrollo, ruralidades, y la división social del trabajo, tanto los análisis de la reproducción social como del mercado de trabajo. 